\title{
Postural Preparation to Stepping: Coupled Center of Pressure Shifts in the Anterior-Posterior and Medio-Lateral Directions
}

\author{
by \\ Clint Hansen ${ }^{1}$, Jacques LaRue ${ }^{2,3}$, Manh-Cuong Do², Mark L. Latash ${ }^{4}$
}

\begin{abstract}
We explored changes in the postural preparation to stepping introduced by modifications of the initial coordinates of the center of pressure (COP). We hypothesized that the postural adjustments in the anterior-posterior direction would persist across all initial COP manipulations while the adjustments in the medio-lateral direction would be highly sensitive to the initial COP coordinate. Healthy subjects stood on a force plate, shifted the body weight to one of the initial conditions that spanned the range of COP coordinates in both directions, and initiated a single step or started to walk. No major changes were observed between the stepping and walking conditions. Changes in the initial COP coordinate in the medio-lateral direction led to scaling of the magnitude of the COP shift in that direction prior to stepping accompanied by a nearly proportional change in the COP shift in the anterior-posterior direction. Changes in the initial COP coordinate in the anterior-posterior direction led to scaling of the magnitude of the COP shift in that direction prior to stepping without consistent changes in the COP shift in the medio-lateral direction. We interpret the results as reflecting a neural organization using a small set of referent body configurations for the postural adjustments.
\end{abstract}

Key words: posture, stepping, center of pressure, biomechanics, referent configuration.

\section{Introduction}

Making a step from a standing posture is accompanied by a consistent pattern of shifts of the center of pressure (COP). In particular, the COP shows a shift backwards with a simultaneous transient shift towards the stepping foot, which is quickly reversed towards the supporting foot (Breniere and Do, 1986; Couillandre et al., 2000; Crenna and Frigo, 1991; Halliday et al., 1998; Jian et al., 1993). Traditionally, postural preparation to stepping is analyzed within the anterior-posterior (AP) and medio-lateral (ML) coordinates. The ML and AP COP shifts have been assumed to play different roles such as generating a moment of force that moves the center of mass (COM) towards the incoming stance foot to help unload the stepping foot (the ML shift) and a moment of force that rotates the body forward (the AP shift).

Several studies have provided evidence for coupling between the AP and ML shifts in preparation to stepping (Azuma et al., 2007; Spencer and van der Meer, 2012). A study of a variety of stepping tasks has shown that a change in the task can lead to an increase in the magnitude of the COP shifts in one of the two directions and to a decrease in the magnitude of

\footnotetext{
1 - Aspetar Orthopaedic and Sports Medicine Hospital, Research Department, Doha, Qatar.

2 - Univ Paris-Sud. UR CIAMS, EA 4532 - Motor Control \& Perception team, Orsay.

3 - UFR STAPS, Université d'Orléans, France.

4 - The Pennsylvania State University, University Park, PA, USA.
} 
the COP shift in the other direction (Degani et al., 2007).

As step and gait initiation depend on the support conditions and entail a shift of the COM position prior to the movement onset (Cau et al., 2014; Massion, 1992), the question arises how they may change due to varying initial conditions. The purpose of this study was thus to examine the effects of different initial COP coordinates on the dynamics of step initiation and walk initiation to explore the coupling between COP shifts in the $\mathrm{AP}$ and $\mathrm{ML}$ directions. We explored coupling between the $\mathrm{AP}$ and $\mathrm{ML}$ COP shifts using variations in the initial COP coordinates and based on earlier studies (Honeine et al., 2012), differences could be expected between COP shifts prior to step and walk initiation; therefore, both tasks were used. We hypothesized that AP and ML COP shifts would be coupled in a sense that changing the initial conditions for one would affect the other.

The ML COP shift may become unnecessary, if the weight of the body has already been placed on the supporting foot during the quiet stance phase. In contrast, the transient COP shift in the AP direction is necessary independently of its initial coordinate; otherwise, no moment of force about the horizontal axis in the frontal plane could be generated. Hence, our second hypothesis was that AP COP shifts would persist across all conditions, while ML COP shifts would disappear in conditions when the stepping leg was sufficiently unloaded. This suggests that COP shifts in the AP and ML direction can be uncoupled by manipulations of the initial posture.

\section{Material and Methods}

\section{Subjects}

Nine healthy volunteers participated in the experiment ( 6 males and 3 females). Their age, body mass and height were $36 \pm 15$ years, $74 \pm 16$ $\mathrm{kg}$ and $1.76 \pm 0.10 \mathrm{~m}$, respectively. All the subjects gave informed consent according to the procedures approved by the Review Board of the Université Paris-Sud.

\section{Methods and Procedures}

A force platform (model BP6001200 AMTI Inc, Watertown, USA) was used to record the forces and moments of force about three axes as illustrated in Figure 1b. A walkway was placed in front of the subject at the level of the platform such that the subject could make at least three normal steps along the walkway. The force/moment signals were collected at $200 \mathrm{~Hz}$ with a 16-bit resolution.

Prior to the experiment, the subject was asked to select a comfortable posture while standing on the platform. That foot position was marked on the platform, and the subject was asked to reproduce it prior to each trial.

There were two parts of the experimental procedure, Step and Walk. Within each part, the subjects performed series of blocked trials $(n=12)$ in eight conditions (Table 1) that differed in the instruction regarding the initial shift of the body weight. The order of conditions and the order of the Step and Walk parts were randomized across subjects. The subjects were always instructed to make the first step with the same foot (right foot in 7 subjects and left foot in 2 subjects). The stepping foot was defined as following: subjects were asked to stand still eyes closed, and a small thrust was applied to their back forcing them to make a step forward. This was repeated three times and the stepping foot was consequently defined as the one that made the steps in response to the unexpected pushes in the back. Subjects were consistent in their response.

In further text, we reversed the right-left coordinates for the two subjects who had initiated stepping with the left foot; hence, the procedure and data are presented as if all subjects initiated stepping with the right foot (left = supporting, right $=$ stepping).

Each trial started with the subject occupying the initial natural standing posture. Then, a verbal instruction was given to shift the body weight according to one of the eight conditions.

The COP shifts were visually controlled by the experimenter using a real time representation of the COP coordinates on-line. The subjects were given 3-4 s to shift the body weight to a new location and stabilize the new posture, and then a verbal command "go" was given. In response to that command, the subject made a self-paced comfortable step forward (Step) or initiated walking forward (Walk) and made 3-4 steps. Each trial lasted for about $8 \mathrm{~s}$, and there were $6 \mathrm{~s}$ intervals between successive trials with 1 min rest intervals between conditions. Two-three practice trials were given prior to data collection in each 
condition.

Standardizing conditions across subjects has no ideal solution so we allowed subjects to select their own initial COP coordinates that, as they perceived, matched the instruction. Hence, the instruction was consistent across subjects while the initial COP locations varied.

\section{Data Processing}

COP shifts were computed using the following approximation:

$\mathrm{COP}_{\mathrm{AP}}=\left(-\mathrm{MML}_{\mathrm{ML}}+\mathrm{F}_{\mathrm{APd}}\right) / \mathrm{FZ}$ and $\mathrm{COPML}^{=}$ $\left(\mathrm{MAP}+\mathrm{F}_{\mathrm{MLd}}\right) / \mathrm{F}_{\mathrm{z}}$

where $\mathrm{F}$ is force, $\mathrm{M}$ is moment of force, $\mathrm{F}_{\mathrm{z}}$ is the vertical component of the ground reaction force, and $\mathrm{d}$ is the distance between the surface of the platform and its origin.

Two characteristics of the COP shift were computed:

1) The COP shifts in the AP and ML directions from the initial position to the one occupied after the COP shift according to the instruction $\left(\triangle \mathrm{COP}_{\mathrm{AP}} \mathrm{IP}\right.$ and $\left.\triangle \mathrm{COP}_{\mathrm{ML}}-\mathrm{IP}\right)$ and over the time interval $\{t 0 ; \mathrm{tEND}\} ; \mathrm{IP}=$ initial position (Figure 1a)

2) The ratio of the COP shifts in the ML and AP directions, $\triangle C O \mathrm{P}_{\mathrm{AP}} / \Delta C O \mathrm{P}_{\mathrm{ML}}$.

Subjects consistently varied the COP coordinates with the instruction in the AP and ML directions (Figure 1c).

\section{Statistical Analysis}

The data are presented as means \pm standard errors. Four ANOVAs with repeated measures were used Condition $\times$ Instruction (Step vs. Walk) on the main outcome variables. In one of the ANOVAs, Condition had four levels that differed in the instruction regarding the initial weight transfer in the ML direction only: CC, C-St, $\mathrm{C}-\mathrm{Su}$, and EX, where St stands for the stepping (right) foot and Su stands for the supporting (left) foot. In the second ANOVA, the factor Condition was split into two factors, Condition-AP regarding the initial weight transfer in the $\mathrm{AP}$ direction (central, forward and backward) and Condition-ML (St and $\mathrm{Su}$ ). To analyze the ratio of the COP shifts $\triangle C O P_{A P} / \triangle C O P M L$ in the ML and AP directions, two ANOVAs (ANOVA-1 \& ANOVA-2) were performed with two factors, Condition-AP (central, forward, backward) and Condition-ML (St and Su). Degrees-of-freedom were corrected in cases of violation of the sphericity assumption. Significant effects were further explored using pairwise contrasts with
Bonferroni corrections. Statistical significance level was set at $\mathrm{p}=0.05$.

\section{Results}

Across all analyses, there were only minimal differences in the COP shift characteristics prior to making a step between the Step and Walk instructions. The factor Instruction (Step vs. Walk) produced little or no effect. The F- and p-values are presented for the Step condition only; similar values were observed for the Walk condition. For the same reasons, the figures illustrate average across the Step and Walk conditions data.

The ANOVAs confirmed significant shifts of both $\mathrm{COP}_{\mathrm{AP}}$ and COPML across different conditions. In particular, the one-way ANOVA with four levels of the factor Condition that differed in the instruction regarding the ML weight shift (CC, C-St, C-Su, and EX), a significant effect was found on $\triangle \mathrm{COPML}(\mathrm{F}[3,8]=142 ; \mathrm{p}<$ 0.0001 ) without an effect on $\triangle C O P_{A P}$.

In the two-way ANOVA, Condition-AP (central, forward, backward) $\times$ Condition-ML (St and $\mathrm{Su}$ ), there were strong significant effects of Condition-AP on $\triangle \mathrm{COP}_{\mathrm{AP}}$ and of Condition-ML on $\triangle \mathrm{COPML}(\mathrm{F}[1,8]>70 ; \mathrm{p}<0.0001)$, without other effects or interactions. Subjects followed the instructions and produced consistent COP shifts in the directions of the instructed body weight shift with only minor effects on COP shifts in the orthogonal direction.

\section{Experimental outcomes}

Changes in the initial COP position in the ML direction led to scaling of the COP shifts during preparation to stepping in both directions when the AP COP coordinate was kept relatively unchanged (effect of Condition: $F[3,8]=33.6$; $p<$ $0.0001)$. These manipulations were associated with smaller, but significant effects on $\triangle \mathrm{COP}_{\mathrm{AP}}$ only in the EX condition when compared to the CC condition (effect of Condition: $F[3,8]=3.04 ; \mathrm{p}<$ 0.05) (Figure 2).

When the initial COP position in the AP direction was modified, it led to major changes in the magnitude of the COP shifts in both AP and ML directions. On average, across the CC, C-St and C-Su conditions, $\triangle C O P_{A P}$ was about $3.2 \mathrm{~cm}$. It increased slightly (not significantly) when the subjects initiated stepping from more anterior 
COP locations (about $3.6 \mathrm{~cm}$ ), and dropped significantly when the stepping was initiated from more posterior COP locations (about $2.0 \mathrm{~cm}$; Figure 1c).

These conditions were selected to form a balanced set with three levels of Condition-AP (Center, Front and Back) and two levels of Condition-ML (St and Su). Two-way ANOVA, Condition-AP $\times$ Condition-ML showed significant effects of both factors on $\triangle \mathrm{COP}$ ML (Condition-AP: $\mathrm{F}[3,8]=3.28 ; \mathrm{p}<0.05$; Condition-ML: $\mathrm{F}[3,8]=68.5$; $\mathrm{p}<0.0001)$. There was also a significant interaction $(\mathrm{F}[3,8]=4.69 ; \mathrm{p}<0.05)$ reflecting the larger differences between the St and Su levels for the forward body weight shift. Analysis of $\triangle \mathrm{COP}_{\mathrm{AP}}$ showed only a significant effect of Condition-AP $(\mathrm{F}[3,8]=4.07 ; \mathrm{p}<0.05)$.

To explore whether the direction of COP shifts was preserved across conditions, we performed analysis of the ratio of the COP shifts in the ML and AP directions. Results of this analysis are illustrated in Figure 3. Note the large effects of the body weight shift in the ML direction (compare the $\mathrm{St}$ and $\mathrm{Su}$ columns in Figure $3 b$ ) with only minor effects of the body weight shift in the AP direction. ANOVA-1 showed a significant effect of the shifts of the body weight in the ML direction on the $\Delta \mathrm{COP}_{\mathrm{AP}} / \triangle \mathrm{COP} \mathrm{ML}$ ratio $(\mathrm{F}[3,8]=7.53 ; \mathrm{p}<0.01)$. These effects were also confirmed by ANOVA-2, which showed a significant effect of Condition$\operatorname{ML}(F[3,8]=26.1 ; p<0.001)$ in the absence of other effects.

The dependence of the ratio $\triangle \mathrm{COP}_{\mathrm{ML}} / \Delta \mathrm{COP}_{\mathrm{AP}}$ on the initial COP shift in the ML direction, but not in the AP direction is illustrated in Figure 4. The Figure presents the data averaged across subjects for the dependences between the initial COP shift in each of the two directions and corresponding values of $\triangle \mathrm{COP} \mathrm{ML}_{\mathrm{M}} / \triangle \mathrm{COP} \mathrm{AP}_{\mathrm{A}}$. Note the strong linear dependence of $\triangle \mathrm{COP}_{\mathrm{ML}} / \triangle \mathrm{COP} \mathrm{PP}_{\mathrm{AP}}$ on the initial COP ML coordinate (black dots, thick regression line, $\mathrm{R}>0.95, \mathrm{p}<0.01$ ) and the lack of such a dependence on the initial AP coordinate (open dots, thin regression line, $\mathrm{R}<0.2)$.

Table 1

The eight experimental conditions related to shift of body weight prior to the movement initiation

\begin{tabular}{|c|c|c|c|}
\hline $\begin{array}{l}\text { Body } \\
\text { Shift }\end{array}$ & Condition & Acronym & Description \\
\hline \multirow{3}{*}{ central } & central-central & $\mathrm{CC}$ & $\begin{array}{c}\text { no shift of the body weight as compared to the natural } \\
\text { quiet stance }\end{array}$ \\
\hline & central-supporting & $\mathrm{C}-\mathrm{Su}$ & $\begin{array}{l}\text { shifting the weight to the left foot without a shift in the } \\
\text { AP direction }\end{array}$ \\
\hline & central-stepping & C-St & $\begin{array}{l}\text { shifting the weight to the right foot without a shift in the } \\
\text { AP direction }\end{array}$ \\
\hline \multirow{2}{*}{ forward } & forward-supporting & F-Su & shifting the weight toward the toes of the left foot \\
\hline & forward-stepping & F-St & shifting the weight toward the toes of the right foot \\
\hline \multirow[t]{3}{*}{ backward } & $\begin{array}{l}\text { backward- } \\
\text { supporting }\end{array}$ & $\mathrm{B}-\mathrm{Su}$ & shifting the weight toward the heel of the left foot \\
\hline & backward-stepping & B-St & shifting the weight toward the heel of the right foot \\
\hline & extreme & EX & $\begin{array}{l}\text { shifting the weight toward the outer edge of the } \\
\text { supporting foot without a shift in the AP direction }\end{array}$ \\
\hline
\end{tabular}



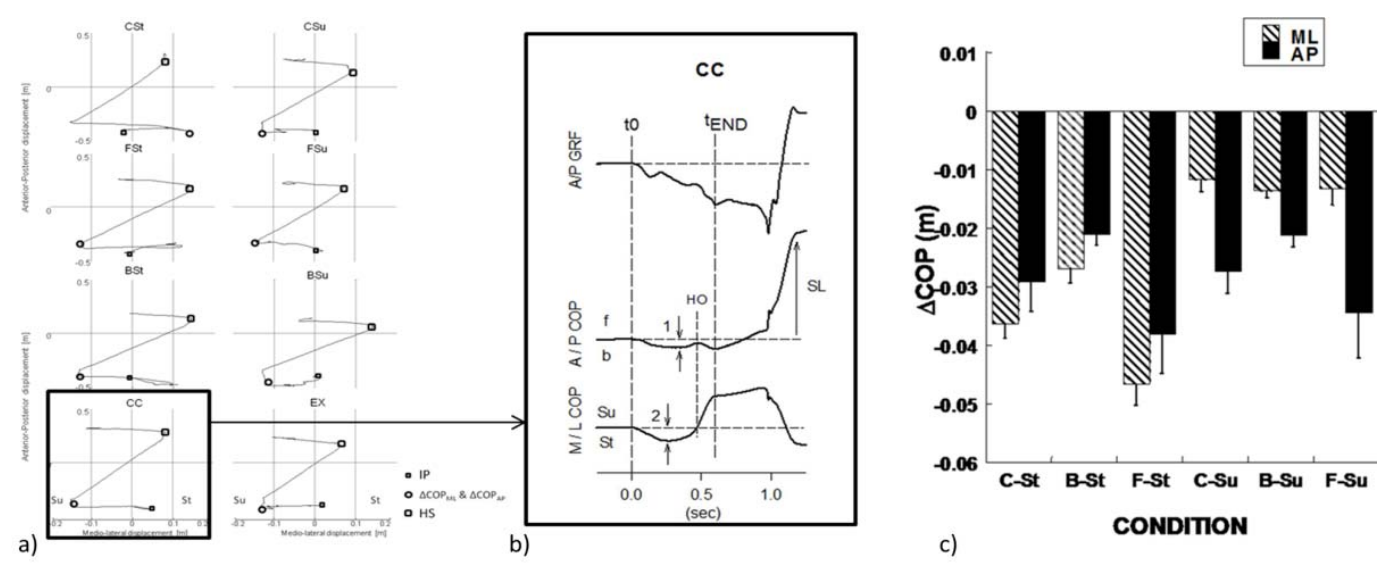

Figure 1a

A typical trial of each stepping condition from one subject. Left part: AP displacement as a function of the ML COP displacements. Right part: AP and ML COP displacements. IP: Initial position before stepping movement; $\triangle C O P_{M L} \mathcal{E} \triangle C O P_{A P}$ : COP shifts in the medio-lateral and anterior-posterior directions prior to the step; HS: Heel strike of the stepping foot; Su, St: support and starting side.

\section{Figure $1 b$}

Figure $1 b$ illustrates a typical time profile of COP shifts in the AP and ML directions by a representative subject. Upper trace: AP ground reaction force. Middle and bottom traces: AP and $M L C O P$ displacements. $t 0$ : onset of stepping movement; $H O$, heel-off of stating foot; $t E N D$ footoff time; SL step length; $f, b$ : forward, backward; Su, St: support and starting side; 1, 2: maxima of

the AP and MLCOP shifts. The time of COP shift initiation ( $\left.t_{0}\right)$ was defined as the earliest deviation from the baseline of $C O P_{A P}$ or COP $M L$. Usually, both $C O P_{A P}$ and $C O P_{M L}$ deviations started simultaneously. The time of the stepping foot take-off ( $\left.t_{E N D}\right)$ was defined when the COPML reached a plateau.

\section{Figure 1c}

Initial COP coordinates under different instructions as compared to the coordinates during natural quiet stance. In the initial conditions, body weight was shifted to the center of the stepping foot (C-St), heel of the stepping foot (B-Su), anterior area of the stepping foot (F-St), center of the supporting foot (C-Su), heel of the supporting foot (B-Su), anterior area of the supporting foot ( $F$ $\mathrm{Su}$ ), and the outer edge of the supporting foot (EX). Averages across subjects and across the two instructions, step and walk, with standard error bars are shown for the medio-lateral $(M L)$ and anterior-posterior (AP) COP shift. 


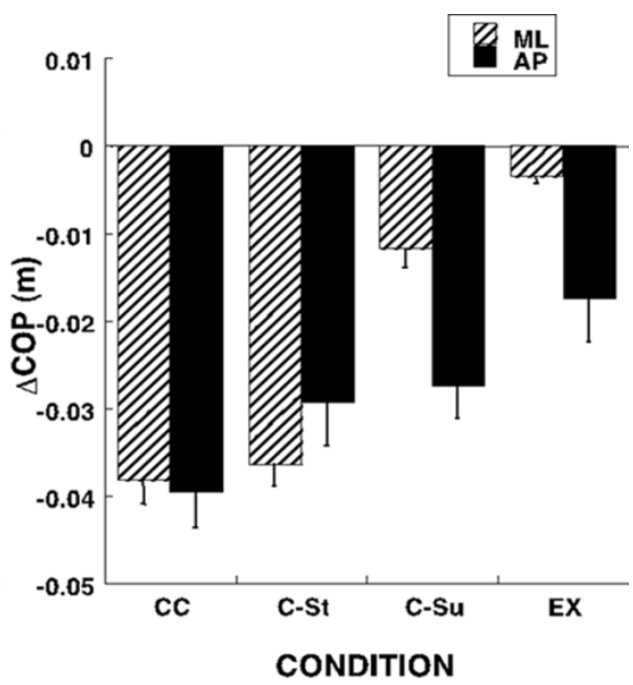

Figure 2

COP shifts ( $\triangle C O P)$ under different instructions regarding the medio-lateral $(M L)$ body weight shift $(C C, C$ $S t, C-S u$, and EX) prior to the initiation of the first step. In the initial conditions, the instruction regarding the body weight coordinate corresponded to the quiet natural stance (CC) or required its shift to the center of the stepping foot (C-St), center of the supporting foot (C-Su), or the outer edge of the supporting foot (EX). Averages across subjects and across the two instructions, step and walk, with standard error bars are shown for the ML and AP COP shift.
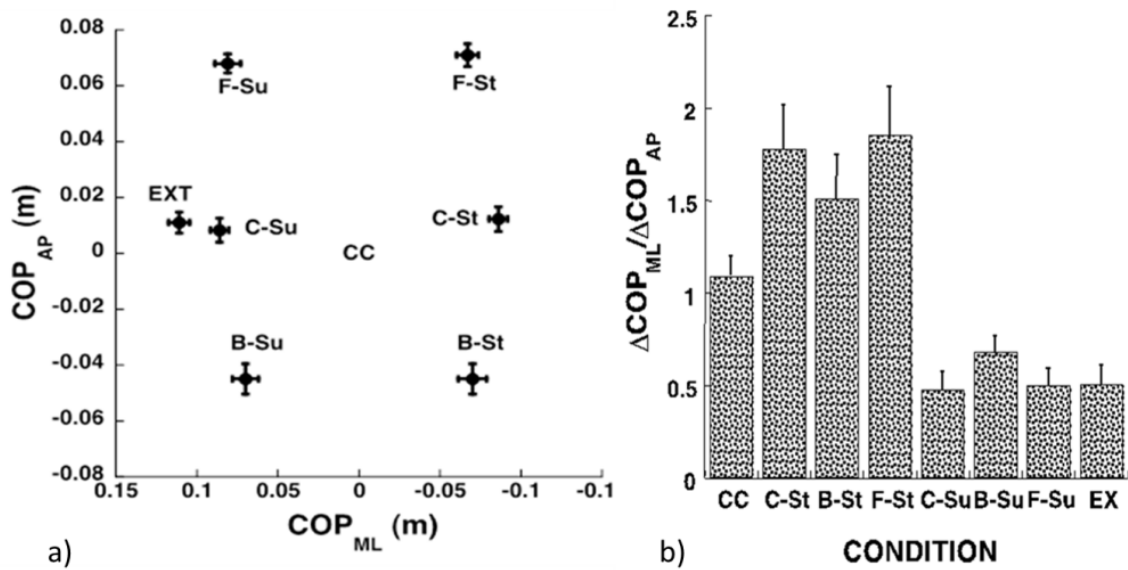

Figure 3a

$C O P$ shifts $(\triangle C O P)$ under different instructions regarding the anterior-posterior $(A P)$ and medio-lateral $(M L)$ body weight shift prior to the initiation of the first step: shift to the center of the stepping foot (C-St), heel of the stepping foot (B-St), frontal area of the stepping foot (F-St), center of the supporting foot (C-Su), heel of the supporting foot (B-Su), or frontal area of the supporting foot (F-Su). Averages across subjects and across the two instructions, step and walk, with standard error bars are shown for the ML and AP COP shift.

\section{Figure $3 b$}

The ratio between the COP shifts in the medio-lateral and anterior-posterior directions $\left(\triangle C O P_{M L} / \triangle C O P_{A P}\right)$ under different instructions: natural quiet stance (CC) or shifted to the center of the stepping foot (C-St), heel of the stepping foot (B-Su), frontal area of the stepping foot (F-St), center of the supporting foot (C-Su), heel of the supporting foot $(B-S u)$, frontal area of the supporting foot (F-Su), or the outer edge of the supporting foot (EX). Averages across subjects and across the two instructions, step and walk, with standard error bars are shown. Note the strong dependence of the ratio on the COP coordinate in the ML direction, but not in the AP direction. 

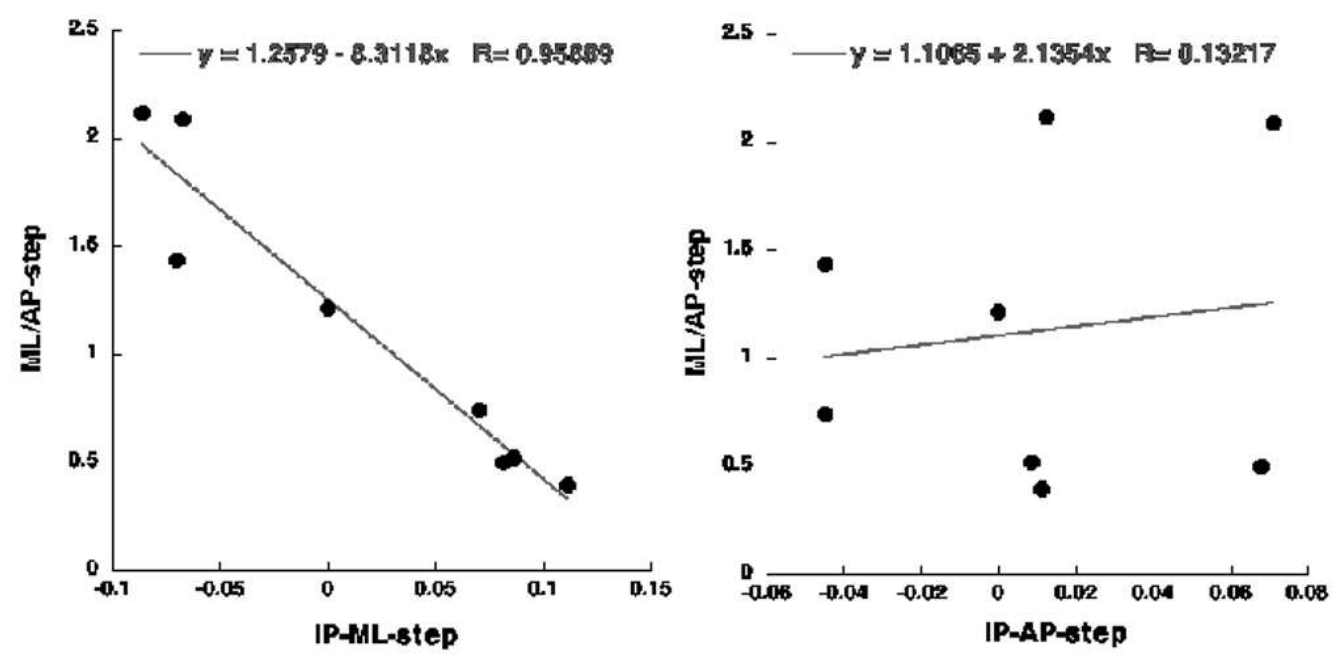

Figure 4

The dependence between the initial COP shifts in the medio-lateral (ML, left plot) and anteriorposterior (AP, right plot) directions and the ratio of the COP shifts in the two directions $\left(\triangle \mathrm{COP} \mathrm{PL}_{\mathrm{ML}} / \triangle \mathrm{COP}_{\mathrm{AP}}\right)$ across all conditions. Each point represents an average across subjects for one of the conditions. Note the strong links between $\triangle \mathrm{COP} \mathrm{ML} / \triangle \mathrm{COP} A \mathrm{P}$ and $\triangle \mathrm{COP}$ in the ML direction $(p<0.01)$, but not in the AP direction. Regression equations are shown.

\section{Discussion}

Both hypotheses formulated in the Introduction received only partial support in the experiment. First, we hypothesized that COP shifts in the AP and ML directions would be coupled in a sense that changing the initial conditions for one would affect the other. The experiment showed only a weak coupling between COP shifts in the two directions. Second, we hypothesized that COP shifts in the AP direction would persist across all conditions, while COP shifts in the ML direction would disappear in conditions when the stepping leg was sufficiently unloaded. In fact, while AP COP shifts persisted across conditions, their magnitude scaled, sometimes without a clear mechanical reason. COP shifts in the ML direction scaled with initial COP ML coordinate, but did not disappear even when nearly the whole weight of the body was initially placed on the supporting foot.

We acknowledge that allowing subjects to select initial COP coordinates matching the instruction best may be a drawback. However, using absolute COP shifts and COP shifts expressed in percent of body height would also raise problems for subjects of different weight.

Postural adjustment to stepping: Superposition of two processes?

The traditional description and analysis of postural preparation to stepping uses shifts of the COP along two axes selected in the body-centered reference frame, AP and ML (Miyasike-daSilva and Mcllroy, 2012). This approach is based on assuming two processes running relatively independently of each other during step preparation, body weight transfer and the generation of a moment of force about the ML axis, MY (Couillandre et al., 2000; Crenna and Frigo, 1991). The former is reflected in COP shifts along the ML axis while the latter is reflected in COP shifts along the AP axis.

Some of our results support this approach. Indeed, changes in the initial COP coordinate along the ML axis had significant effects on the COP shift along this axis during 
step preparation (Figures 2 and 3). On the other hand, changes in the initial COP AP coordinate by themselves did not directly change the requirements for the MY production. Nevertheless, we observed significant changes in the COP shift along the AP axis with changes in the initial COP AP coordinate (Figure 3). These patterns were consistent across postural preparations to making a step and initiating the gait, which is not trivial given earlier reports on differences in the postural preparation patterns to the step and gait (Honeine et al., 2013).

Taken together, our observations do not provide consistent support for the idea of a superposition of two processes organized about the two main body axes during postural preparation to stepping. The findings of a significant coupling between the COP shifts along the two axes suggest that the neural processes involved in postural preparation may be organized not about the AP and ML axes, but involve a different coordinate system.

\section{Coupling of COP shifts in the AP and ML directions}

Coupling between the COP shifts in the $\mathrm{AP}$ and ML directions was observed under manipulations of the initial COP ML coordinate, which led to significant, close to proportional changes in the COP shifts (Figures 2 and 3). For a fixed instruction with respect to the initial COP ML coordinate, variations of the initial COP AP coordinate showed no significant coupling between the COP shifts in the AP and ML directions. We can think of two main reasons for the coupling, mechanical and neural.

The mechanical reason is that the directions of action of many leg and trunk muscles are not parallel to the AP and ML axes. As a result, scaling of the muscle activation levels with changes in the initial conditions could be expected to lead to correlated COP shifts in the $\mathrm{AP}$ and ML directions. This factor, however, is expected to lead to parallel adjustments in COP shifts in the two directions no matter what the original cause for the adjustments is. The fact that we observed correlated COP shifts in the two directions only under manipulations of the initial ML coordinate, but not AP coordinate, speaks against a crucial role of the mechanical factor.

During whole-body actions, muscles are commonly united into groups with close to parallel scaling of activation levels within each group (d'Avella and Bizzi, 2005; Tresch and Jarc, 2009). Such groups have been addressed as synergies (Ivanenko et al., 2004; Ting and Macpherson, 2005) and as muscle modes (DannaDos-Santos et al., 2007; Krishnamoorthy et al., 2003a, 2003b). The latter term implies that such muscle groupings represent reflections of higherorder neural variables that may be further united into synergies stabilizing important features of performance (Latash, 2010; Latash et al., 2007). It has been assumed that muscle groups are formed based on everyday experience in such tasks as standing and walking (Dominici et al., 2011; Lacquaniti et al., 2012; Safavynia et al., 2011). Using muscle modes and relatively simple scaling rules of their involvement may be the reason for the observed patterns of parallel scaling in COP shifts in the AP and ML directions.

\section{Interpretation within the referent configuration hypothesis}

According to the referent configuration (RC) hypothesis, the CNS uses neural variables associated with subthreshold depolarization of neuronal pools to modify referent values for important task-related variables addressed as RC (Feldman, 2009; Feldman and Levin, 1995). The differences between the actual and referent coordinates lead to non-zero signals that give rise to a cascade of few-to-many mappings ultimately resulting in activation of numerous muscles that contribute to the task execution (Latash, 2010). At each stage of such a hierarchy, redundant sets of the output elemental variables show co-variation that stabilizes their combined effect.

With respect to whole-body tasks, the repertoire of postural adjustments can result from a relatively small set of scalable shifts of RCs resulting in relatively small sets of muscle groups, three to five, that show parallel activations as those assumed in the notion of muscle modes (Robert et al., 2008). This interpretation is consistent with the idea of a few eigenmovements forming the kinematic patterns of postural adjustments (Alexandrov et al., 2001) including those typical of the ankle strategy and hip strategy (Diener et al., 1988).

The observed parallel change in $\triangle \mathrm{COP}_{\mathrm{AP}}$ and $\triangle C_{\text {COP }}$ under the manipulations of the initial COPmL coordinate (Figure 4) may be a reflection of a learned direction of shifts of the 
body RC in the external space.

The parallel scaling of the COP shifts in the $\mathrm{AP}$ and $\mathrm{ML}$ directions, suggests that a standard shift of the body RC is used and scaled in magnitude across the conditions. This was true, however, only for manipulations of the ML COP coordinate in the initial posture, not during manipulations of the AP COP coordinate (Figure 4). At the moment, we can offer only a speculative interpretation for these contrasting results. Prolonged standing is associated with larger COP excursions in the ML direction compared to the AP direction (Duarte and Zatsiorsky, 1999): people more frequently shift the body weight from one foot to the other than rock forward and backwards. It is possible, therefore, that even extreme shifts of the initial COP in the ML direction allowed the subjects to use their learned shifts of the RCs associated with step initiation resulting in parallel scaling of the postural adjustments in the two directions. Shifting the body weight forward or backwards might force the subjects to perform the task in a qualitatively different way leading to de-coupling of the AP and ML COP shifts.

\section{Practical Implications}

Our findings have potential implications for motor learning and, consequently, sports. In particular, step initiation from postures with different initial COP coordinates is common across many sports such as basketball, football, etc. The parallel scaling of the COP shifts in the $\mathrm{AP}$ and ML directions observed in our study could be used to optimize posture prior to movement initiation in a variety of sports, e.g. in soccer when trying to deflect a player from the other team. Learning to react to extreme shifts of the COP may also be beneficial in sport, and specific training could optimize COP shifts in such conditions.

\section{References}

Alexandrov A, Frolov A, Massion J. Biomechanical analysis of movement strategies in human forward trunk bending. I.Modeling.Biological Cybernetics, 2010; 84: 425-434

Azuma T, Ito T, Yamashita N. Effects of changing the initial horizontal location of the center of mass on the anticipatory postural adjustments and task performance associated with step initiation. Gait Posture, 2007; 26(4): 526-531

Breniere Y, Do M. When and how does steady state gait movement induced from upright posture begin? J Biomech, 1986; 19: 1035-1040

Cau N, Cimolin V, Galli M, Precilios H, Tacchini E, Santovito C, Capodalgio P. Center of pressure displacements during gait initiation in individuals with obesity. J Neuroeng Rehab, 2014; (11): 82

Couillandre A, Breniere Y, Maton B. Is human gait initiation program affected by a reduction of the postural basis? Neurosci.Lett, 2000; 285(2): 150-154

Crenna P, Frigo C. A motor programme for the initiation of forward-oriented movements in humans. $J$ Physiol, 1991; 437: 635-653

Danna-Dos-Santos A, Zatsiorsky V, Latash M. Muscle modes and synergies during voluntary body sway. Exp Brain Res, 2007; 179: 533-550

d'Avella A, Bizzi E. Shared and specific muscle synergies in natural motor behaviors. Proc. of the National Academy of Sciences USA, 2005; 102: 3076-3081

Degani A, Danna-Dos-Santos A, Latash M. Postural preparation to making a step: Is there a "motor program" for postural preparation? J App Biomech, 2007; 23: 261-274

Diener H, Horak F, Nashner L. Influence of stimulus parameters on human postural responses. J.Neurophysiol, 1988; 59(6): 1888-1905

Dominici N, Ivanenko Y, CappelliniG, d'Avella A, Mondi V, Cicchese M, Lacquaniti F. Locomotor primitives in newborn babies and their development. Science, 2011; 334: 997-999

Duarte M, Zatsiorsky V. Patterns of center of presure migration during prolonged unconstrained standing. 
Motor Control, 1999; 3(1): 12-27

Feldman A. Origin and advances of the equilibrium-point hypothesis. Adv.Exp.Med Biol, 2009; 629: 637-643

Feldman A, Levin M. Positional frames of reference in motor control: their origin and use. Behav Brain Sci, 1995; 18: 723-806

Halliday SE, Winter DA, Frank JS, Patla AE, Prince F. The initiation of gait in young, elderly, and Parkinson's disease subjects. Gait Posture, 1998; 8: 8-14

Honeine J, Schieppati M, Gagey O, Do M. The functional role of the triceps surae muscle during human locomotion. PlosOne, 2013; 8: e5294

Ivanenko Y, Poppele RE, Lacquaniti F. Five basic muscle activation patterns account for muscle activity during human locomotion. J Physiol, 2004; 556: 267-282

Jian Y, Winter D, Ishac M, Gilchrist L. Trajectory of the body COG and COP during the initiation and termination of gait. Gait Posture, 1993; 1: 9-22

Krishnamoorthy V, Goodman S, Latash M, Zatsiorsky V. Muscle synergies during shifts of the center of pressure by standing persons: Identification of muscle modes. Biol Cybern, 2003; 89: 152-161

Krishnamoorthy V, Latash ML, Scholz JP, Zatsiorsky V. Muscle synergies during shifts of the center of pressure by standing persons. ExpBrain Res, 2003; 152: 281-292

Lacquaniti F, Ivanenko Y, Zago M. Patterned control of human locomotion. J of Physiol, 2012; 590: 2189-2199

Latash M. Motor synergies and the equilibrium-point hypothesis. Motor Control, 2010; 14: 294-322

Latash M, Scholz JP, Schöne G. Toward a new theory of motor synergies. Motor Control, 2007; 11(3): 276-308

Massion J. Movement, posture and equilibrium: interaction and coordination. Prog Neurobiol, 1992; 38: 35-56

Miyasike-daSilva V, Mcllroy W. Does it really matter where you look when walking on stairs? Insights from a dual-task study. PlosOne, 2012; 7(9): e44722

Robert T, Zatsiorsky V, Latash M. Multi-muscle synergies in an unusual postural task: Quick shear force production. Exp Brain Res, 2008; 18: 237-253

Safavynia S, Torres-Oviedo G, Ting L. Muscle Synergies: Implications for Clinical Evaluation and Rehabilitation of Movement. Top Spinal Cord Inj Rehabil, 2011; 17(1):16-24

Spencer LM, van der Meer AL. TauG-guidance of dynamic balance control during gait initiation across adulthood. Gait Posture, 2012; 36(3): 523-526

Ting L, Macpherson J. A limited set of muscle synergies for force control during a postural task. J. Neurophysiol, 2005; 93(1): 609-613

Tresch M, Jarc A. The case for and against muscle synergies. Curr Opin Neurobiol, 2009; (19): 601-607

\section{Corresponding author:}

\section{Clint Hansen}

Aspetar Orthopaedic and Sports Medicine Hospital, Research Department, Doha, Qatar

E-mail: Hansen.Clint@gmail.com 